\title{
LA EDUCACIÓN POPULAR EN LOS ESTADOS UNIDOS: LAS HUELLAS DE PAULO FREIRE Y MYLES HORTON
}

Roberto Elisalde ${ }^{1}$

RESUMEN: El presente trabajo esta situado en el campo de la historia y la educación. Busca reconstruir uno de los procesos menos transitados por la tradición de la educación popular latinoamericana, y norteamericana. En este caso rescatar vínculos, similitudes, encuentros y diferencias de dos concepciones y referentes de la educación popular: Paulo Freire y Myles Horton. Freire durante su largo exilio de los años 60 y 70 fue invitado en reiteradas oportunidades a los Estados Unidos. Allí llevo a cabo intercambios en los que expuso y debatió con pares norteamericanos acerca de las posibilidades de interpretar y "aplicar" las ideas de "Pedagogía del oprimido "en territorios del primer mundo". Harvard, Massachusetts, Tennessee, Los Ángeles fueron las principales estadías de Freire. En algunos casos con breves recorridos y en otros, con la meta de retornar para completar trabajos comunes. Así sucedió con el histórico educador popular norteamericano Myles Horton, creador de uno de los movimientos más relevantes en la historia de la educación popular de los EEUU, el Centro Highlander de Teennesse. Era corriente que a tanto a Horton como a Freire en sus presentaciones los participantes, indistinatmente, le preguntaran: “¿es posible pensar la educación popular en los Estados Unidos?" ¿Los países del Primer mundo pueden implementar estrategias de "tradición popular" en sus organizaciones y en sus aulas? Precisamente estas inquietudes, y sobre todo, la reconstrucción histórica de estos encuentros serán el eje central de este trabajo.

\section{POPULAR EDUCATION IN THE UNITED STATES: THE FOOTPRINTS OF PAULO FREIRE AND MYLES HORTON}

ABSTRACT: This work is located in the field of history and education. It seeks to reconstruct one of the processes least traveled by the tradition of Latin American and North American popular education. In this case, to rescue links, similarities, encounters and differences of two conceptions

\footnotetext{
${ }^{1}$ Doutor em História pela Universidade Nacional de La Plata (UNLP). Pós-Doutor em Educação pela Universidade Estadual Júlio de Mesquita Filho (UNESP) campus de Marília. Professor e investigador da Universidade de Buenos Aires-UBA. Integrante da Rede de Investigadores e Organizações Sociais da América Latina-RIOSAL-CLACSO-CTA-A. Email: relisalde@hotmail.com
} 
and referents of popular education: Paulo Freire and Myles Horton. Freire, during his long exile in the 1960s and 1970s, was repeatedly invited to the United States. There I carried out exchanges in which he presented and debated with North American peers about the possibilities of interpreting and "applying" the ideas of "Pedagogy of the oppressed" in first world territories ". Harvard, Massachusetts, Tennessee, Los Angeles were Freire's main stays. In some cases with short tours and in others, with the goal of returning to complete common work. This was the case with the historic American popular educator Myles Horton, creator of one of the most relevant movements in the history of American popular education, the Teennesse Highlander Center. It was common for both Horton and Freire in their presentations to be asked indiscriminately, "Is it possible to think popular education in the United States?" Can First World countries implement "popular tradition" strategies in their organizations and in their classrooms? Precisely these concerns, and above all, the historical reconstruction of these meetings will be the central axis of this work.

Key words: Popular Education, Freire, Horton

"Entre las formas que van hacia la sierpe

Entre las formas que van hacia el cristal

Dejaré caer mis cabellos..."

Federico García Lorca, Poeta en Nueva York, 1940.

\section{INTRODUCCIÓN}

La vida y la producción de Paulo Freire han sido tratadas de manera extendida en el campo de la historia de la educación, en particular en Latinoamérica, sin embargo, son menos difundidos sus viajes a otros países, especialmente los que realizó a los Estados Unidos.

Exilio, invitaciones académicas, encuentros con referentes norteamericanos de la educación popular o simplemente convites por trabajo constituyeron la multiplicidad de motivaciones que llevaron al legendario educador a emprender un simpar recorrido por el país del norte. Como al poeta Federico García Lorca y a muchos otros notables humanistas de Iberoamérica, el contraste entre sus propios valores y sensibilidades con la materialidad exuberante del imperio les provocó un sinnúmero de reacciones y consecuencias. Algunas de ellas fueron simplemente declaraciones de índole cultural o estrictamente política o incluso, la producción de libros, artículos, agua fuertes o documentales testimoniales de aquella experiencia. 
En el caso de Paulo Freire, su capacidad transformadora, sus inquietudes militantes, lejos de abatirlo, como al poeta andaluz, lo llevaron a buscar rastros y evidencias de la existencia de "otra Historia de los Estados Unidos", tal como lo señalaba el notable historiador norteamericano Howard Zinn².

Sus presentaciones y encuentros se produjeran en torno a seminarios, talleres e intercambios en los que expuso y debatió con referentes norteamericanos acerca de las posibilidades de interpretar y "aplicar" las ideas de "Pedagogía del oprimido "en territorios del primer mundo. Harvard, Massachusetts, Tennessee, Los Angeles fueron las principales estadías de Freire en Estados Unidos. En algunos casos con breves recorridos y en otros, con la meta de retornar para completar encuentros y trabajos, así sucedió con el histórico educador popular norteamericano Myles Horton.

Horton fue el creador de uno de los movimientos más relevantes en la historia de la educación popular norteamericana: el Centro Highlander de Teennesse. Como veremos más adelante, un verdadero territorio irradiador de ideas, acciones e hitos políticos que nos permitirán establecer relaciones y particularidades con la tradición freiriana Latinoamericana.

Era corriente que tanto a Horton y como a Freire en sus charlas, diálogos, encuentros o intercambios le formularan la pregunta: “¿es posible pensar la educación popular en los Estados Unidos?" ¿Los países del Primer mundo pueden implementar estrategias de "tradición popular" en sus organizaciones y en sus aulas? Precisamente estas inquietudes, y sobre todo, la reconstrucción histórica de estos encuentros serán el eje central de este trabajo.

\section{FREIRE: LOS COMIENZOS DE UN LARGO EXILIO Y SUS VIAJES A LOS ESTADOS UNIDOS}

Los años iniciales de Freire como activo educador surgen desde su participación en el movimiento de cultura popular de Recife y en la realización de trabajos de alfabetización con sectores sociales postergados desde 1963. Precisamente las tareas educativas que llevó a cabo en esa región fueron tenidas en cuenta por el antropólogo

\footnotetext{
${ }^{2}$ Zinn, Howard (1999) La otra historia de los Estados Unidos, Ed. Otras voces. Buenos Aires.
} 
Darcy Ribeiro -en ese entonces ministro de educación-, quien lo convocó para aplicar su método de enseñanza a través de la preparación de un Plan de alcance nacional. La politicidad de la educación, el compromiso social y la liberación conformaban sus principios más sentidos, de tal modo que su modalidad alfabetizadora llevaría necesariamente esa impronta:

“Freire creó un método de alfabetización que en la medida en que los iletrados aprendían a leer y escribir iban dialogando sobre problemas de su realidad y buscando alternativas para transformarlos" ${ }^{3}$

Los antecedentes de estas iniciativas alfabetizadoras se remiten a 1962 y 1963 . En esos años la USAID y la SUDENE ${ }^{4}$ establecieron un convenio con el estado de Rio Grande do Norte para financiar un programa de educación de adultos y de expansión de la enseñanza primaria. Más de 37 millones de dólares fueron invertidos en ese plan. El secretario de educación de ese estado le pidió a Paulo Freire que organice el flamante programa de educación de la SEDENE. Freire aceptó pero con dos condiciones: que el estado realice un acuerdo con la Universidad Estatal de Recife en lugar de hacerlo con él, y que los estudiantes de la Universidad de Rio Grande do Norte participen del proyecto. En 1963 y comienzos de 1964, las incipientes técnicas de alfabetización freiriana fueron prontamente utilizadas en los estados de Alagoas y Rio Grande do Norte. Unas 4000 personas se inscribieron en los cursos.

El Programa de la misión de SEDENE-USAID en Recife, mientras tanto, presentaba algunos problemas en su desarrollo. Una de ellas era el faltante de material escrito que se había solicitado en el inicio de los cursos. La otra razón era de índole política, la politización -propia del método Freire- comenzó a molestar a los fazendeiros y coroneles,

\footnotetext{
${ }^{3}$ Gadotti, Moacir, Paulo Freire. Uma biografia, (2001),Cortez editora/Unesco, Brasil, pág. 6.

${ }^{4}$ El programa de USAID (Agencia de los Estados Unidos para el Desarrollo Internacional) fue un organismo creado para promover, según afirmaban sus creadores, el intercambio y la promoción de áreas básicas como la educación en países latinoamericanos. En 1963 se firmó en Brasil un convenio entre USAID y el organismo estatal brasileño denominado SEDENE (Superintendencia de desarrollo del Nordeste). El mismo fue impulsado por el economista Celso Furtado. Este tipo de acuerdos formaron parte de las políticas de corte desarrollista desplegadas por los Estados Unidos en el hemisferio. Fueron estrategias de promoción y control para la región que sumadas a las intervenciones militares indirectas (o directas en algunos casos) caracterizaron la política exterior norteamericana del período. El final del Programa USAID-SEDENE de Rio Grande do Norte y Recife, sumado al golpe militar del 64 parecen dar evidencia de esta modalidad de intromisión y dominación hemisférica.
} 
es decir a los grandes propietarios de las zonas rurales. Muchos de los estudiantes que participaban del programa eran de izquierda y comenzaban a ser mal vistos por los grupos de poder, tanto de los sectores gubernamentales como los eclesiásticos.

En un mundo jerárquico y paternalista como el del nordeste, la palabra de los coroneles era ley, de allí que promover el pensamiento crítico entre los campesinos no tuvo una buena acogida en la sociedad de aquel entonces. De allí que en enero de 1964 se produjo un desenlace previsible: la insatisfacción con el método pedagógico Freire y la preocupación con los contenidos políticos del programa llevaron a la USAID a quitar el apoyo financiero del programa, justo tres meses antes del golpe militar contra el presidente J.Goulart ${ }^{5}$.

A raíz de esta situación, Freire debió exiliarse, refugiándose por breve tiempo en Bolivia, para luego instalarse por cuatro años en Chile ${ }^{6}$. Allí sistematizó buena parte de las ideas y prácticas que estaba desarrollando territorialmente con sus equipos y que formaban parte de sus planes de alfabetización. Al poco tiempo se publicaron sus libros Educación como práctica de la libertad (1965) y Pedagogía del oprimido (1969); en ellos presentaba sus concepciones acerca de la "conciencia dominada", la "opresión y alienación social y educativa", la "concientización", la "dialoguicidad" y la "relación entre educación, política y liberación". La perspectiva de Freire se fundaba en la praxis; proponía una acción que reflexione críticamente y una reflexión crítica diseñada por la práctica y validada en ella. El vínculo estrecho entre autoconciencia y acción conforman una "dinámica permanente y constante de nuestra actitud hacia la cultura misma"7 Es precisamente en el nivel de la praxis donde transcurre la concientización del término, dado que la concientización implica una "inserción crítica en la historia", es decir, un compromiso y una toma de conciencia voluntaria, personal e histórica que tiene como meta la transformación del mundo. Freire interpreta el quehacer humano como praxis y reflexión, teoría y praxis.

\footnotetext{
${ }^{5}$ Ver Jerome Levinson y Juan Onis (1970) The Alliance That Lost Its Way, Chicago, Quadrangle Books.

${ }^{6}$ En Chile va a estar cuatro años, hasta 1969, luego retorna a este país en tiempos de la Unidad Popular. Allí realizará tareas de alfabetización en áreas rurales, trabajando con grupos de capacitación relacionados con la reforma agraria y participar de numerosos cursos en la Universidad.

${ }^{7}$ Freire, Paulo, Pedagogía de la ciudad, Ed. Siglo XXI, México, 1995
} 
"La delimitación de sus objetivos y de sus métodos y la determinación de su fuerza de acción vienen dadas por la teoría que lo informa; es el marco teórico que informa las modalidades de acción liberadora y opresora. Surge entonces la teoría de la acción liberadora desde una matriz dialógica tendiente a conseguir la humanización de los hombres; promueve una auténtica formación valórica que genere un auténtico cambio cultural". 8

Sus textos van a estar influidos por numerosos autores e ideas pertenecientes a diversas corrientes de la teoría social y política de aquellos años, tales como la teología de la liberación, el marxismo en sus múltiples vertientes y la psicología de Erich Fromm ${ }^{9}$. Es posible encontrar en sus escritos, sobre todo en Pedagogía del Oprimido, una destacada presencia de conceptos e ideas del maoísmo, el pensamiento y acción de Ernesto Che Guevara, así como evidentes lecturas de los Cuadernos de Cárcel de Antonio Gramsci o el propio estadounidense Myles Horton, como luego veremos. También es factible percibir concepciones y rasgos de referentes a las luchas por la liberación del Tercer Mundo, como ocurre con Franz Fanon ${ }^{10}$ y Amilcar Cabral ${ }^{11}$.

Ya en 1970, Paulo Freire iniciaba la segunda etapa de su exilio y se trasladaba a Estados Unidos, Europa y África. En cada uno de esos lugares desempeñó tareas que iban a redundarle un fuerte apoyo de las organizaciones populares e incluso, como en África (Tanzania, Angola, Guinea Bissau, Cabo Verde) permitirle llevar a cabo sus ideas

\footnotetext{
${ }^{8}$ Gadotti, M op. cit, pág. 11.

${ }^{9}$ La opresión a la que Freire hace mención en sus escritos afecta los aspectos más profundos de la persona, en la medida en que por la socialización hemos incorporado a nuestro bagaje psicológico. Para desarrollar esta idea, Freire recurre a un enfoque de corte "freudomarxista", fuertemente influenciado por Erich Fromm y su concepción del "miedo a la libertad". También acuerda con la crítica de Fromm a la cultura y escuela impregnadas de rechazo a la vida.

10 Franz Fanon fue un escritor y militante argelino que luchó por la liberación de su país frente a la dominación colonial francesa en la década del ' 50 y ' 60 . Una de sus obras más reconocidas e influyentes fue Los condenados de la tierra. Existen numerosos trabajos que establecen relaciones e influencias del pensamiento freiriano y el del argelino. Ver: Martins, Pablo, "Confluencias entre el pensamiento de Franz Fanon y Paulo Freire", en Revista Educação, Santa María, v.37, n.2, p.241-256, maio/ago.2012.

${ }^{11}$ Amílcar Cabral fue uno de los dirigentes de Guinea Bissau más destacados en la lucha de liberación nacional y social contra el colonialismo europeo. En 1975 Freire fue invitado por el gobierno revolucionario para llevar adelante un Plan de Alfabetización. En 1977 publica Cartas a Guinea Bissau. Apuntes de una experiencia pedagógica en proceso en el que recupera aquella experiencia, a la luz de sus logros y complicaciones que supo tener ese proceso.
} 
alfabetizadoras de la mano de gobiernos revolucionarios que se reivindicaban como parte de un proceso regional emancipatorio.

Su primer viaje a Estados Unidos lo realizó en los inicios de 1970, trabajando casi un ciclo lectivo en Harvard, luego viajó a Ginebra donde completó sus 16 años de exilio. ${ }^{12}$ Por estos años, la noción de educación popular iba a configurarse en el cuestionamiento al carácter injusto del orden social de tipo capitalista propio de las sociedades latinoamericanas. Este diagnostico estructural de la educación popular freiriana fue nutriéndose con el aporte e influencia de las ciencias sociales críticas (especialmente el marxismo), el auge de las luchas populares y de la izquierda de los años sesenta y setenta. Freire era convocado e invocado como referente y denunciante de los rasgos opresivos y antipopulares del sistema educativo y los programas de alfabetización oficiales. De allí su caracterización de los sistemas educativos predominantes como opresores y/o de formación "bancaria"13. El calificativo de "popular" por parte de la educación popular no tenia que ver propiamente con el sujeto colectivo de sus accioneslas clases desposeídas/dominadas-, sino con el horizonte político del cambio ${ }^{14}$. Se enfatizaba, además, que no todas las experiencias y programas educativos cuyo destinatario era el pueblo tenían la intencionalidad emancipatoria propia de la educación popular. $^{15}$

El elemento identificatorio de la educación popular es, entonces, su explicita intencionalidad política, expresada al servicio de los intereses del pueblo y en la construcción del socialismo latinoamericano y democrático, como le gustaba afirmar:

\footnotetext{
${ }^{12}$ En 1974 Freire va a participar también en la Argentina como asesor del Plan de Alfabetización DINEACREAR, desarrollado durante el gobierno peronista de Héctor Cámpora y que planteaba la necesidad de llevar a cabo un Plan educativo que diera cuenta de las necesidades de los trabajadores en el marco de un proyecto de "liberación nacional y social". En Homenaje a la CREAR. Ministerio de Educación. Buenos Aires, 2009.

13 El "termino educación bancaria" será una expresión de cuño freiriana y fuertemente popularizada. Con ella se refería a la promoción de sujetos pasivos que proponían los sistemas educativos, en los que se "depositaban" conocimientos sin desarrollar o incorporar los saberes de los educandos.

${ }^{14}$ Torres, Alfonso, "Prácticas de conocimiento en Educación Popular", en Revista Encuentro de saberes, Año I, N¹, pág 54.

15 "La educación popular se plantea entonces, como un esfuerzo en el sentido de la movilización y de la organización de las clases populares con vistas a la creación de un poder popular" "Entrevista a Paulo Freire", en Torres, Rosa Maria, Educación Popular. Un encuentro con Paulo Freire, CEAL, Buenos Aires, 1985.
} 
“Me parece urgente que superemos un sinnúmero de preconceptos contra la democracia, asociada siempre a la burguesía. Al abrirnos a su nombre muchos pensamos en conservadurismo, explotación burguesa, socialdemocracia. Yo pienso en socialismo. ¿Por qué no? ¿Por qué no conciliar transformación social, profunda, radical, con libertad? ${ }^{16}$

Sus viajes por el mundo y el reconocimiento de experiencias de educación popular con fuertes anclajes territoriales, no solo reafirmaron la verosimilitud de estas luchas, incluso para el Primer mundo, sino también lo llevaron a Freire al encuentro, intercambio y confluencia de saberes con destacados representantes y organizaciones sociales que luchaban desde la educación, por un mundo diferente. Tal fue el caso, como veremos, de su encuentro en los Estados Unidos con Myles Horton del Centro Highlander Folk School en Cumberland Plateau en Tennessee, por cierto, una experiencia poco conocida para los latinoamericanos.

De este proceso se desprenden varios temas de interés para la historia de la educación popular de la región: ¿Cuál fue la tarea del Centro Highlander, y sobre todo, qué implicancias tuvo para la educación y la política norteamericana desde los años '30? ¿Cuáles fueron los tópicos centrales del encuentro Horton-Freire y ¿Cuál fue su relevancia para la tradición de la educación popular en los Estados Unidos?

\section{MYLES HORTON Y EL CENTRO DE EDUCACIÓN E INVESTIGACIÓN HIGHLANDER: LA LUCHA CONTRA LA SEGREGACIÓN SOCIAL Y POR DERECHO AL VOTO}

En los años treinta Myles Horton y un grupo de colaboradores inicia su experiencia en el Centro de Educación e Investigación Highlander. Parte de su iniciativa tuvo como objetivo dar cuenta de las necesidades formativas de la población obrera de la región de Tennessee en el sur de los Estado Unidos. Allí comienza organizando espacios de intercambio y formación junto a jóvenes voluntarios. Durante los periodos iniciales, Highlander se vinculó con organizaciones sindicales y sociales, a los que proveyó de cursos formativos y talleres de estudio. Muchos grupos sindicales - como el ClO (Congress for Industrial Organizations), la Iglesia Católica, el Partido Comunista, el Partido Socialista-

\footnotetext{
${ }^{16}$ Freire, P y Faúndez, A, Por uma pedagogia da pregunta, Río de Janeiro, Paz e Terra, 1985, pág. 76.
} 
estaban durante esa época creando escuelas populares. La organización impulsada por Horton fue parte de este proceso. Con el correr de los años fueron incrementándose los espacios formativos, así como las aspiraciones organizacionales del Centro.

Fue también por esos años que Horton decidió viajar a Dinamarca para aprender del reconocido circuito de educación popular existente en los países nórdicos, probablemente los numerosos grupos migratorios daneses llegados a los Estados Unidos desde Europa habían generado esos circuitos de influencias e intercambio de experiencias.

“Todos llegamos a la conclusión que teníamos que comenzar a aprender de las personas que habían realizado tareas semejantes a las nuestras. Aprender junto a ellos y adaptarlos a nuestras necesidades y condiciones. Esto fue lo que hicimos en Dinamarca"17

En Dinamarca estuvieron en las llamadas "escuelas del pueblo" y quedaron impresionados con lo que allí se había realizado. Horton investigó sobre esas experiencias, previo a su viaje, en la Universidad de Chicago:

"Yo no buscaba una técnica o un método...Estaba buscando un proceso acerca de cómo relacionarme con las personas...Finalmente lo encontré, un lugar en las montañas en donde promover la educación para adultos"18

Con el correr de los años fueron creados una serie de talleres en Highlander sobre el problema de la segregación en el Sur. De este proceso surgió la llamada Escuela ciudadana. La misma había sido referida por primera vez en una conferencia en las Naciones Unidas. Esau Jenkins, un miembro de la comunidad negra de Johns Island, Carolina del Sur, fue a Highlander con Septima Clarck, una profesora que había visto a Highlander de Charleston, también de Carolina del Sur. Jenkins dijo en ese encuentro internacional que le parecía óptimo hablar sobre el mundo y sus problemas, pero que tenía profundas dificultades en su país. Su dificultad era cómo conseguir ayuda para enseñar a las personas a leer y aprobar el registro exigido para electores en condiciones de votar. Ese examen era administrado por funcionarios blancos que no tenían el menor deseo de que los negros tuviesen derecho al voto, y usaban la restricción del

\footnotetext{
${ }^{17}$ Myles Horton sobre acontecimientos de 1935, En Freire, P y Horton M, (2011) O camino se faz caminando, Editora Vozes.

${ }^{18}$ Horton, 1975. Ibid. Pág. 35.
} 
analfabetismo como un medio para impedírselos. Él afirmaba que venía intentando enseñar a las personas a leer cuando viajaban en ómnibus, puesto que había un servicio de transporte que traía empleados domésticos y operarios de fábrica, sus vecinos negros, para el trabajo. En el bus el tenia un público cautivo e intentaba enseñarles algo de lectura durante el viaje. Era un líder reconocido en aquella ciudad ya que se ocupaba de los problemas del pueblo, además del analfabetismo, también estaba atento a reclamar y organizar a la comunidad negra desempleada.

\section{PROGRAMAS PÚBLICOS DE ALFABETIZACIÓN Y REFERENTES EN HIGHLANDER}

Desde principios del siglo $X X$, los programas o grupos de alfabetización que existían en Estados Unidos y en particular en la región de Highlander al sur de los Estados Unidos eran de pésima calidad y con escasa participación de maestros, cuando no expulsivos.Esta realidad abarcaba no sólo a las poblaciones empobrecidas, sino también a buena parte de los inmigrantes ${ }^{19}$. Los estudiantes abandonaban rápidamente sus cursos, había poco interés: ni los profesores ni los alumnos estaban motivados, a pesar de que contaban con fondos de los gobiernos federales y estaduales dirigidos a la alfabetización.

Era claro que había un problema y era bastante simple. Los alfabetizadores no estaban tratando a las personas con el mínimo respeto. El tipo de programa que estaban ofreciendo era visto por los estudiantes como un insulto. Las personas adultas mayores tenían que sentarse en bancos pequeños para niños. Los pequeños reían y los llamaban "abuelos de piernas largas". Esto indudablemente generaba desmotivación en ellos, a la vez que alimentaba la falta de respeto y de especial atención que necesitaban para continuar sus estudios.

"Por eso el programa que organizaríamos -afirmaba el Centro- tenía que tomar como base el respeto a las personas. Finalmente decidimos que no tendríamos profesores

\footnotetext{
${ }^{19}$ Es interesante la carta que Charles Nagel, funcionario del gobierno del presidente norteamericano William Talf - y político formado en el estado sureño de St Louis- le envía al propio mandatario con la finalidad de poner en evidencia los problemas expulsivos que generaban en 1913 los Programas alfabetizadores oficiales: "El gobierno de su país los desalienta en su intento de aprender a leer y escribir su lengua, y estos inmigrantes que llegan a nuestras costas luchan verdaderamente por liberarse de las condiciones en que han sido obligados a vivir." En Nuñez García, Silvia y Zermeño Padilla, Guillermo (1988), EUA: Documentos de su historia política, T.III, Ed. Instituto Mora, México, pág.552.
} 
formados, ninguno que hubiese sido capacitado para ser profesor. Los profesores formados pensaban -al menos en aquellos tiempos- identificaban a los adultos analfabetos como niños analfabetos". ${ }^{20}$

El otro problema que había era el trabajo con personas blancas, "ellos siempre quieren predodominar sobre las personas negras", afirmaban los organizadores de Highlander. En el Centro se pensó que ese problema simplemente podía eliminarse no contando con profesores blancos, y fue así como se hizo al principio. La otra cosa que se discutió en los inicios fue que las personas debían aprender rápidamente a leer ya que tenían poquísimo tiempo para hacerlo. Debían contar con material pedagógicamente adecuado para la población joven y adulta con la que se iba a trabajar. Los textos para el registro de votantes poseían ciertas dificultades que exigían un trabajo de mayor intensidad y compresión en la lectura y la escritura.

Una de las primeras profesoras que marcó el proyecto Highlander fue Bernice Robinson, era una joven afrodescendiente que había ido a visitar a el Centro y quedó impresionada con el trabajo social y educativo desarrollado. Ella no había completado el curso secundario pero tenía una gran disposición a aprender y a enseñar. Rápidamente se ofreció como colaboradora y estableció un excelente trato con las personas que iban a alfabetizarse. Bernice comenzó a enseñar sin ningún plan especial. Ella era lo opuesto a Septima, otra de las primeras colaboradoras que poseía formación educativa y pedagógica: "yo no soy profesora -afirmaba Bernice-, realmente no sé porque ellos quieren que haga esto, pero estoy aquí y aprenderé con ustedes. Iré aprendiendo a medida que vayamos caminando" ${ }^{21}$. Comenzó a trabajar con algunas cartillas como por ejemplo la declaración de los derechos humanos; era una estrategia algo compleja para una etapa de iniciación pero supo que con ese tipo de desafíos los estudiantes se mostraban aún más motivados. En aquella época Highlander tenía una declaración de objetivos que explicaba qué era la institución y cuáles sus fines; ese material también fue aplicado para los trabajo de lectura. Bernice pensó que esa declaración contenía algunas ideas buenas sobre la democracia y la ciudadanía, y también pidió una copia de ese

\footnotetext{
${ }^{20}$ https://www.highlandercenter.org/our-story/

${ }^{21}$ Cita en Freire, Horton, op.cit. pág.74.
} 
material para trabajar. Ese era el nivel de materiales que usó en sus aulas, aunque la mayoría de las veces lo que hacían los maestros -y Bernice en especial- era animarlos a practicar y escribir sus nombres, domicilio, facturas de pago y otros elementos vinculados a su vida cotidiana. Los estudiantes prontamente comenzaron a intentar presentarse a las pruebas de admisión para el voto, y como nos recuerdan los integrantes del centro, naturalmente, algunos lo lograron y otros debieron esperar más tiempo.

\section{EDUCACIÓN POPULAR, ORGANIZACIÓN COMUNITARIA Y NUEVAS ESCUELAS CIUDADANAS}

Lo que les iba quedando claro a los integrantes de la experiencia del Centro Highlander era que las instancias de estudio no solo eran clases de alfabetización, sino que constituían parte de un proceso de organización comunitaria y popular. A medida que pasaba el tiempo, comenzaban a hablar de las cosas que harían cuando consiguiesen el voto, se referían a cómo usar su ciudadanía y la importancia que comenzaban a darle a su escuela ciudadana. Todo eso ayudaba a motivarlos; el examen consistía en la realización de sus escritos frente al tribunal de registro de lectores. Cuando el consejo responsable por el registro decía que ya tenían derecho a votar, el Centro consideraba que ya había aprobado el curso de alfabetización, ese era su examen final.

Durante los años 50 y 60, más del $80 \%$ consiguieron pasar esa prueba. Al poco tiempo de la apertura de la primera escuela comenzaron a solicitarnos abrir otras nuevas. Bernice coordinó esas clases, e inmediatamente necesitaron incorporar más profesores. La propia Berenice comenzó a preparar a los nuevos profesores y el Centro organizó la expansión de las Escuelas Ciudadanas. Numerosas regiones y ciudades las reclamaron:

“Establecimos una especie de programa de formación para profesores para la Escuela de Ciudadanía en el propio Highlander. Bernice quedó al frente de ella y Séptima era la consultora". ${ }^{22}$

\footnotetext{
${ }^{22}$ Horton, 1964. En https://www.highlandercenter.org/our-story
} 
De todo el proceso la única persona que había recibido algún tipo de formación en educación era Séptima, que era la directora de todo el proyecto. Esa fue la estructura en la cual se establecieron las escuelas. El programa de "formador de formadores" como tal comenzó en enero de 1957 y en 1961 ya habían sido capacitados más de 400 profesores y más de 4 mil alumnos pasaron por sus cursos de alfabetización. Los electores -en condiciones de votar- en ésta área habían aumentado en un $300 \%$. La idea era que Highlander fuera una institución que promoviera la transformación multiplicada en clave de organización popular de la comunidad; este proceso por aquellos años parecía alcanzarse. El programa fue enriqueciéndose y siendo más eficiente a medida que se desarrolló. Highlander y las Escuelas ciudadanas eran, a la vez, un formato de organización comunitaria difundido con altos niveles de autonomía. Los centros nuevos que iban surgiendo no necesariamente dependían de Highlander. Era un programa de bajo costo, se contaba con becas o algunos recursos para pagar a los profesores y profesoras, aunque no había salarios. Se financiaba la capacitación, pero no las clases que los docentes daban, la comunidad quedaba como responsable de ello. Por lo tanto, ninguno de los profesores cobraba por el trabajo, eran todos voluntarios-militantes del Centro, "personas negras enseñando a otras personas negras", afirmaban los miembros de las comunidades participantes. La organización se expandió tan rápidamente que debió reorganizar su estructura inicial. Por eso fueron creados varios programas más junto a la colaboración de sindicatos y organizaciones sociales de trabajadores:

“En esa época trajimos a Andrew Young a Highlander -que más tarde fue embajador de los Estados Unidos en las Naciones Unidas y luego prefecto de Atlanta- para coordinar la expansión del programa. Antes de su llegada, Martin Luther King preguntó si podíamos elaborar un programa para Southern Christian Leadership Conference (SCLC). Septima siempre le hablaba acerca del programa de Escuelas de Ciudadanía. Al principio no creíamos que ese fuera el mejor programa para la Conferencia, pero luego King quedó interesado, por ello lo aplicamos para la SCLC."23 Ese vínculo consolidó la masividad del Centro entre la comunidad afrodescendiente, especialmente de los estados del Sur.

\footnotetext{
${ }^{23}$ Horton, 1962, Ibid.
} 
Durante esos años, el movimiento de Derechos Civiles ya había comenzado. Paso de Montgomery para Atlanta y la idea comenzaba a expandirse. Las Escuelas de Ciudadanía pasaron a ser el programa de la Conferencia; fueron hechas algunas adaptaciones pero el programa permaneció más o menos igual. Andy Young y otras personas lo consideraron como esencial para el movimiento de Derechos Civiles. El programa fue muy reconocido en un momento en que ningún otro plan de alfabetización estaba siendo aplicado en los Estados Unidos.

"En esa época costaba tanto enseñarle a alguien a leer y a escribir como mandarlos a Harvard por un año, nosotros hacíamos un trabajo por lo menos de 100 u\$ por persona en términos de costos reales. Esto era realizado en un periodo medio de tres meses con dos extensas clases por semana, la noche con cerca de 75 a $80 \%$ personas que lo completaban y que podían registrarse como lectores. No hay duda que funcionó. Funcionó y se expandió." ${ }^{24}$.

Myles Horton se refería al trabajo de sus colaboradores/as y compañeros/as como parte de las necesarias estrategias militantes de aquellos años. La solidaridad, el compromiso y el trabajo pedagógico adecuado lo expresaban las mujeres que fueron parte de la creación y diseño de la experiencia. Tal fue el caso de su mención al trabajo de Bernice:

"En aquellos años Bernice no intentaba hacer que todos la siguieran, o que todos leyeran juntos o separadamente. Ella no usaba el tipo de método que todos tenían que estar haciendo la misma actividad al mismo tiempo. Hacían lo que les parecía interesante. Ella decía que al final todos querían aprender a leer porque como algunos aprendían, los otros también querían hacerlo. Por eso Bernice se concentraba en aquellos que querían aprender, ellos aprendían el máximo que podían de la cartilla y después los otros se involucraban. No era siempre la misma actividad la que debía ser hecha, o el mismo tema para una clase. La próxima vez sería otra cosa. Aprender a leer la cartilla estaba

\footnotetext{
${ }^{24}$ Horton, 1965,op.cit.
} 
combinando con aprender a leer y escribir el nombre con el llenado de órdenes de pagos y un poco de cada cosa." 25

Luego de ese proceso formativo, las personas fueron capaces de votar. Esau Jenkings dijo "vamos a tener un programa para el segundo estadio", necesitamos dar una continuidad a las escuelas de alfabetización, serán las escuelas de ciudadanía. Tenemos que ayudar a las personas a entender que ellas pueden usar sus votos de una manera inteligente y conseguir que se interesen en candidatearse en alguna actividad. También tenemos que hablar acerca de cómo vamos a usar nuestro poder si lo obtuviéramos: escuelas, salud, trabajo, afirmaban "necesitamos hablar sobre la lucha más general por la justicia".

El movimiento de los derechos civiles estaba comenzando a tomar forma y Esau quería ser parte de él. Hay un estudio hecho de éste programa por Carl Tjerandsen -secretario ejecutivo de una fundación- que aportó algún dinero y escribió sobre el programa, su estudio tenía detalles de la propuesta del segundo estadio de formación.

La educación popular, comúnmente, en las cruzadas de alfabetizadoras era un paso adelante, tenía un objetivo, leer y escribir, aunque para el Centro no era necesariamente la meta. La meta era convertirse en ciudadanos críticos, en sujetos políticos. Por eso cuando la clase podía leer y escribir, los maestros iban en busca otras cosas; los estudiantes tenían que continuar leyendo y escribiendo sobre textos y libros que el segundo estadio iba a exigir que tuvieran en cuenta.

"La enseñanza termina al cabo de un tiempo y la educación comunitaria comienza. Fueron los negros que denominaron al proyecto "como de escuela", fueron ellos los que llamaron a las personas como "profesor", la llamaron Escuela de Ciudadanía y tenían un profesor. Para ellos era la verdadera educación, era su terminología, no la mía ni la de Bernice."²6 Recuerda Horton que a inicios de los 70, la dirección estadual de educación de adultos, encargada del programa de alfabetización, le solicitó hacer talleres en Highlander para hablar sobre la Escuela de Ciudadanía, como un ejemplo de la utilización del abordaje educativo en grupos en lugar de un abordaje individualista. El Centro decidió que los programas en Tennessee sean hechos en grupos y no con tutores individuales; el estado

\footnotetext{
${ }^{25}$ Horton, 1980, Ibid.

${ }^{26}$ En Freire-Horton, op.cit, pág. 87.
} 
estaba buscando ayuda para conseguir que las personas cambien la enseñanza individual hacia un trabajo basado en el progreso grupal.

"Nosotros teníamos nuestros propios problemas y desafíos, realmente no estábamos trabajando en una situación verdaderamente revolucionaria como aquellos procesos de Cuba, o Nicaragua en los '80. Pero tampoco teníamos metas como que la gente solo pretenda, por ejemplo leer y escribir para conseguir un empleo, aquí tenemos otras aspiraciones, como poder votar, construir ciudadanía crítica"27.

Horton solía afirmar que no veía en Estados Unidos una campaña nacional que valorice los problemas de la alfabetización, muchos gobiernos -afirmaba- intentaban lanzar campañas sin expresar una razón para ello, eran emprendimientos parciales sin mucho compromiso. “Es posible encontrar focos en el país donde podríamos tener programas de alfabetización bien organizados, esto podría ocurrir en realidad en cualquier lugar y en todas las regiones, de todos modos, entiendo que los pobres y las personas que no saben leer y escribir tienen una sensación de que sin cambios estructurales no vale la pena quedar entusiasmados con nada; saben mucho más claramente que los intelectuales, que las reformas no reforman, no cambian nada. Ellos ya fueron cobayos para demasiados programas. Pero se puede llegar hasta ellos con una idea radical -como la que pudimos vincular al programa de las Escuelas de Ciudadanía - donde vieron algo significativo; se transformarían en ciudadanos del mundo. Entonces se identificarían con esa idea, pero no con objetivos limitados y de corto plazo que no los llevan a ninguna parte"28

Con el tiempo, las Escuelas ciudadanas de Highlander fueron incorporadas regularmente a las Conferencia de Liderazgos Cristianos de sur que lideró originalmente Luther King. Myles Horton trabajó con su equipo para construir esos Programas de educación popular en otras partes del Sur profundo norteamericano. Años después el Centro Highlander fue pionero en la realización de talleres, conferencias y acciones en clave de "formación de formadores" por todo Estados Unidos.

\footnotetext{
${ }^{27}$ En Freire-Horton, op.cit. Las permanentes referencias a los problemas con el voto se fundamenta en que en los Estados Unidos el voto no es obligatorio, y para votar, era necesario inscribirse y estar alfabetizado. ${ }^{28}$ Freire-Horton, op. cit, pág.84.
} 
En la actualidad el Centro continúa con su trabajo, especialmente en la región de los Apalaches. Y además de la formación de las comunidades postergadas han sumado a su caudal temático de trabajo, problemas medioambientales, justicia económica, pobreza, fortaleciendo el trabajo y liderazgo de los jóvenes ${ }^{29}$.

\section{ENCUENTRO AMERICANO ENTRE EDUCADORES POPULARES: PAULO FREIRE Y MYLES HORTON EN TENNESSEE}

Ya señalamos que la primera visita de Freire a Estados Unidos le significó la posibilidad de radicarse un tiempo como profesor visitante en la Universidad de Harvard, pero que al poco tiempo, optó por otras propuestas de trabajo y partió a Ginebra con nuevas iniciativas ${ }^{30}$. Debemos trasladarnos hacia la década de los ' 80 para dar cuenta de los nuevos derroteros de Freire en Norteamérica.

Como es sabido, esos años se caracterizaron por el fuerte avance del neoliberalismo, en toda la región. La aplicación de las recetas neoliberales de los técnicos en economía provenientes de la Universidad de Chicago significaron para América Latina un fuerte proceso de precarización social y endeudamiento económico. Entre los años 80 y principios del nuevo milenio, la implementación de políticas neoliberales basadas en un modelo de apertura económica y en la aplicación de medidas de corte monetarista significaron para Latinoamérica la profundización de las desigualdades existentes: aumento de la desocupación, deterioro de los salarios, flexibilización/ precarización laboral e incremento de los índices de pobreza; como consecuencia de ello un creciente sector de la población quedó excluida del acceso al empleo, al consumo e incluso a la educación. $^{31}$

\footnotetext{
${ }^{29}$ Para consultar las actividades que en la actualidad desarrolla el Centro Highlander: https://www.highlandercenter.org/

30 "Entre el inicio de enero y mediados de febrero de 1973, Freire recorre doce estados diferentes de los Estados Unidos, realizando un número elevado de encuentros con trabajadores y trabajadoras sociales, educadoras y educadores, sobre Pedagogía del oprimido". En Kohan, Walter (2019), Paulo Freire mas que nunca. Una biografía filosófica, CLACSO.

31“(En la Argentina), la implementación de un modo de acumulación flexible produjo una estructuración diferente del mercado de trabajo, reflejada en la multiplicación de las formas de contratación (empleo autónomo, tercerización, subcontratación, trabajos temporarios)...El costo laboral en la década del '90, bajó un 62\%, según las estadística oficiales del Ministerio de Trabajo. Por otro lado se observó también un
} 
Precisamente en el plano educativo la aplicación de las reformas tuvo un carácter global. El proceso de transformación educativa, como lo llamaron sus gestores, estuvo enmarcado en un contexto en el que la función del estado era redefinida a partir de los principios básicos de la ideología monetarista.32 El estado se reorganizó internamente y se convirtió, al mismo tiempo, en el responsable de la conducción general del proceso económico, social y político. El debilitamiento de la estructura estatal se llevó a cabo a través de la privatización de las empresas nacionales y de la desregulación de los servicios públicos. En el marco de predominio de estas ideas se implementaron reformas educativas en la mayor parte de América Latina -los países del Conosur fueron en su totalidad cubiertos por estas medidas, constituyéndose Chile y luego Argentina en los referentes máximos del neoliberalismo en la región-.

"El contexto en el que se desarrollaron las reformas educativas fue el proceso de globalización, el cual está caracterizado por la profundización del desarrollo capitalista, la consolidación y la ampliación de las desigualdades estructurales. Estas políticas fueron, entre otras, la apertura económica indiscriminada tanto del comercio como de la libre circulación de las divisas internacionales." 33

La educación fue cuestionada como derecho social y se la planteó como un servicio en el que el conocimiento adquirió un carácter mercantil. Se la pensó como una dimensión regida por las leyes de la economía de mercado, dejando librada las posibilidades de estudiar a cada sujeto en su situación social individual. La descentralización financiera y administrativa del sistema educativo y la centralización

notorio aumento del empleo no registrado (en negro), que pasó del 26,5\% en 1990, al 35\% en 1999, y del subempleo, que en el 2001 alcanzaba el 16,3\% (Indec)", en Svampa, M., La sociedad excluyente, La Argentina bajo el signo del neoliberalismo, Editorial Taurus, Buenos Aires, 2005, pág. 42.

32 "El amplio uso del mercado reduce la sobrecarga que sufre el entramado social, ya que todas las actividades que abarca hace innecesaria la conformidad. Cuanto más amplio sea el número de actividades cubiertas por el mercado, menos será el número de cuestiones en las que se requieran decisiones expresamente políticas..." Fridman, Milton, Capitalismo y libertad, Madrid, 1996, cap.2, pág.41.

${ }^{33}$ Zibechi, Raúl (2003), Genealogía de la revuelta. Argentina: La sociedad en movimiento. Editorial Letra Libre, Buenos Aires. 
pedagógica ejercida "desde afuera" a través de los sistemas de evaluación se presentaron como claves del nuevo esquema. ${ }^{34}$

En ese contexto hemisférico, Paulo Freire acepta una invitación para viajar a dar algunas conferencias e intercambios en los Estados Unidos. En particular, se destacan dos encuentros que el brasileño tuvo con referentes de la educación norteamericana. El primero fue con el académico Ira Shor, durante su estancia en Massachusetts durante 1985. Y el segundo evento fue en diciembre de 1987, en Tennessee, con el educador popular Myles Horton.

Ambos diálogos mas tarde fueron publicados, el primero en español y al inglés, y el segundo, sólo en portugués y español. El encuentro con Shor, publicado bajo el nombre de Freire-Shor. Miedo y Osadía ${ }^{35}$ reviste un destacado y diferenciado interés en la tradición de la educación popular, ya que en este caso, su análisis gira en torno a la naturaleza del sistema educativo norteamericano. Conceptos como "cultura del silencio", "racismo", "sexismo" y las posibles "potencialidades liberadoras de sus escuelas" constituyen parte del amplio menú de temas abordados por ambos educadores, anticipando para el propio Freire muchas de las problemáticas que deberá tratar en 1989 cuando asuma el cargo de Secretario de Educación pública en San Pablo, bajo el gobierno municipal del Partido de los Trabajadores.

El encuentro con Myles Horton marcó un hito en las relaciones entre dos educadores populares de larga trayectoria y plasmó la confluencia de las trayectorias de la Educación Popular Latinoamericana y la estadounidense ${ }^{36}$. Las dos figuras expresaban un manifiesto compromiso de por vida en la lucha por la transformación radical, especialmente en el campo de la educación para jóvenes y adultos. Myles Horton y Paulo Freire tenían mucho en común. A lo largo de su trabajo ambos subrayaron el carácter claramente político de la actividad educativa, insistiendo en que no puede haber ninguna

\footnotetext{
${ }^{34}$ En nuestro país, como en otros países de América Latina, este proceso privatista y mercatilizador tuvo un punto de inicio y a la vez de inflexión desde los años de las dictaduras militares durante la década del ' 70 . En CTERA, CNTE, y otros (2005), Las reformas educativas en los países del Cono Sur, Buenos Aires, CLACSO, págs.46-47

${ }^{35}$ Freire, Paulo y Shor (2014), Ira, Miedo y osadía, Ed. Siglo XXI, Argentina.

${ }^{36}$ Freire-Horton, op.cit.
} 
educación 'neutral', diseñado sus estrategias de formación en el marco de una disputa constante para la generación de relaciones sociales radicalmente democráticas.

La vida de Myles Horton fue claramente menos prolífica como escritor que Freire y posiblemente es por este motivo que su contribución a la transformación radical educación de jóvenes y adultos no le ha otorgado el reconocimiento internacional que merece.

Las similitudes entre el pensamiento de Miles Horton y Paulo Freire los llevaron a considerar que las democracias debían ser construidas desde las bases populares. El punto central en el proceso de despertar de la conciencia -decía Freire y coincidía Hortonocurre cuando el oprimido comienza a reconocer su propia dignidad. También reafirmaban que la cooperación ofrece a los oprimidos una gran posibilidad para llegar al control de su propia vida y conquistar la voz que necesitan para organizar un futuro justo para todos ${ }^{37}$.

Los dos pensaban que la verdadera liberación debía ser realizada a través de la participación popular. Y entendían que estas ideas no eran abstracciones, ellas crecían a partir de las luchas y la unión entre la teoría y la práctica.

Como educadores ambos sustentan un abordaje centrado en el estudiante, enfatizando la discusión, el dialogo, la comunicación y respetando el conocimiento de los estudiantes y su capacidad para sumir su propio aprendizaje. Como humanistas Freire y Horton trabajaron en diferentes escenarios, pero ambos se dedicaron a mejorar las situaciones de pobreza en las que vivían grandes poblaciones del mundo. Ambos veían en la educación una fuerza capaz de fortalecer la lucha contra la opresión.

Un análisis de sus filosofías y metodologías ofrece una síntesis heurística de las características de la educación centrada en el estudiante. La manera con la cual ellos aplican ese proceso de educación problematizadora tiene significativa importancia para la implementación de ideas humanísticas que lleven a alcanzar el cambio social ${ }^{38}$.

\footnotetext{
${ }^{37}$ Freire-Horton, op.cit, pág.95.

${ }^{38}$ Conti, Gary, Community College Review N ${ }^{\circ} 5,1977$.
} 
Horton y Freire también reflexionaron en su encuentro sobre sus experiencias formativas, delineando los principales aportes en el desarrollo de sus ideas y prácticas pedagógicas. La conversación ocupó un buen tiempo para hablar sobre la aparición de las escuelas de ciudadanía en caso de Horton y el temprano trabajo de alfabetización de Freire en Brasil. También recorrieron las influencias teológicas y filosóficas de Myles Horton, en particular el trabajo de Reinhold Niebuhr ${ }^{39}$ y sus recuerdos en su estancia por Dinamarca donde entró en contacto con las ideas del obispo Grundtvig y, probablemente, con los principios de Kristen Kold, ideas que se asocian con el concepto de Folk High School ${ }^{40}$.

A Horton le gustaba enfatizar que el Centro Highlander Folk School on the Cumberland Plateau en Tennessee, desde 1932, siempre trabajo fuera de las universidades y de instituciones gubernamentales y que era un centro independiente dedicado a la educación de jóvenes adultos, con especial dedicación para promover una democracia de base. Myles fue perseguido e investigado durante la época del anticomunismo acérrimo de Joseph McCarthy en los años 50 por su activa participación en el movimiento de lucha por los derechos civiles.

Una de las razones que movieron a Paulo Freire a encontrarse con Myles Horton fue, según le gustaba afirmar al educador brasileño, que estaba cansado de que le preguntaran si sus ideas solo eran posibles para países del Tercer mundo. Frente a ello, Freire decía "No", "la historia de Myles y Highlander Center muestra que las ideas de la educación popular también eran aplicadas en el Primer mundo". Ambos coincidían en la necesidad de pensar la educación en clave de politicidad. Rechazaban los "falsos objetivismos y neutralismos". Así lo destaca el investigador Walter Kohan:

"La educación no puede ser apolítica -afirmaba Freire-, políticamente neutra o aséptica. En este punto, concuerda con otro gran educador del siglo XX, Myles Horton (Freire y

\footnotetext{
${ }^{39}$ Niebuhr fue un teólogo y politólogo norteamericano. Es considerado el representante de una corriente denominada "realismo cristiano", y se destacan sus críticas sobre el mito del "progreso indefinido de los Estados Unidos".

${ }^{40}$ La mutua influencia de las ideas nórdicas de educación popular (folkbuilding) no pertenecen sólo a la experiencia de Myles Horton. El propio Freire visitó Suecia y Dinamarca en los años 70 y su presencia e influencia es evidente en las cien escuelas populares existentes en la actualidad en ese país. Ver Elisalde, R., Las escuelas populares en Suecia. De Grundvig a Paulo Freire, Informe presentado en la "Pedagogical Movement: Popular High Schools for Youth and Adults in Argentina, 2001-2011", en the ICAE World Assembly, Malmo-Suecia, 14 - 17 June 2011.
} 
Horton, 1990, 2018). Horton expresa lo que comparte con Freire de la siguiente manera: "neutralidad es simplemente seguir la multitud. Neutralidad es tan solo ser lo que el sistema nos pide que seamos. Neutralidad, en otras palabras, es un acto inmoral” ${ }^{41}$

Finalmente, a lo largo de su prolífico diálogo, los dos educadores legaron saberes y caminos comunes, e incluso destacaron diferencias enriquecedoras para el debate actual sobre las relaciones entre la educación popular y la pública estatal.

Ante el dilema si existía la posibilidad de construir una educación liberadora en el sistema de educación público-estatal, cada uno de ellos se posicionó de manera distinta. Freire, argumento la pertinencia del desafío, señalando, como dice Henry Giroux, un lenguaje de la crítica" de las relaciones de poder existentes en cuanto "lenguaje de la posibilidad" para crear una nueva sociedad de una nueva prácticas social y educacional.

Horton y su equipo de los Apalaches, coincidía con Freire en las metas liberadoras, pero entendía que el sistema público estatal en su país limitaba y subordinaba el espíritu liberador de la educación popular. Highlander buscaba caracterizaba al sistema formal de educación estatal, así como otras instituciones sociales formales, de manera claramente peyorativa y regresiva frente al cambio. Su mensaje era que las prácticas sociales transformadoras son más probables que ocurran fuera del sistema institucional: "Llegamos a la conclusión que las reformas dentro del sistema refuerzan al propio sistema, terminan cooptando a las fuerzas de la transformación. ${ }^{42}$ Esta postura explica su enfoque claramente alternativo sobre la educación popular de jóvenes y adultos.

Freire enfatizaba que es bueno considerar participar en la acción colectiva transformadora tanto fuera como dentro del sistema, y que no debe perderse la oportunidad de trabajar dentro del mismo. Esta parece haber sido la filosofía a lo largo de su vida, pero especialmente a partir de los años 80 , como un educador popular de jóvenes y adultos. Su trabajo en 1989 como Secretario de educación en el Gobierno Municipal de San Pablo reafirmó sus pensamientos, ya que en esa experiencia desarrollará sus tareas 'dentro' del sistema, pero en conjunto con actores que operaban fuera del sistema: los movimientos sociales.

\footnotetext{
${ }^{41}$ Kohan, Walter (2019), Paulo Freire mas que nunca. Una biografía filosófica, CLACSO, pág. 216.

${ }^{42}$ Freire-Horton, op.cit. pág. 120.
} 
A pesar de estas diferencias de punto de vista, pareció no haber ninguna tensión de fondo en los múltiples momentos que tuvo la extensa conversación, más bien al contrario, trataban de buscar coincidencias y argumentar con claridad, sus diferencias. Tres días después de este encuentro, Myles Horton, con 84 años, entró en coma -su salud ya estaba algo deteriorada- y morirá a los pocos días. Freire, por su parte, a poco de retornar a Brasil se encontró con el triunfo del Partido de los Trabajadores en San Pablo, entre otras tantas ciudades. Le aguardaba una difícil tarea. Por primera el legendario educador popular iba a asumir en la gestión pública la Secretaría de la educación del municipio paulista. Tenía por delante nada menos que el desafío de construir "el camino hacia una escuela pública y popular" ${ }^{23}$.

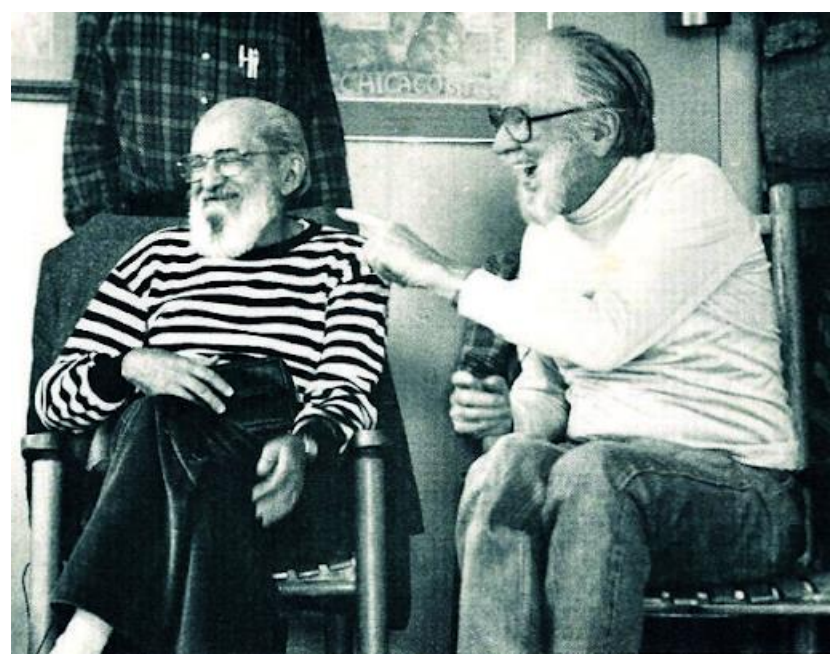

Freire y Horton en Tennessee, 1984 (Foto Archivo Instituto Paulo Freire)

\section{BIBLIOGRAFÍA}

ADAMS, Frank. Highlander Folk School: obtener información, regresar y enseñarl en Harvard Educational Review, Vol. 42, núm. 4, noviembre, 1972.

CONTI, Gary. Community College Review . n5, 1975.

CTERA, CNTE, y otros. Las reformas educativas en los países del Cono Sur, Buenos Aires: CLACSO, 2005. p. 46-47

\footnotetext{
${ }^{43}$ Documentos de la Secretaría de Educación del Municipio de S. Pablo". En Elisalde (2019), Roberto, Paulo Freire, Educación popular, Estado y movimientos sociales, Ed. Biblos, 2019. 2da. Ed.
} 
TORRES, Rosa Maria, Educación Popular. Un encuentro con Paulo Freire, CEAL, Buenos Aires, 1985.

ELISALDE, Roberto. Paulo Freire: Educación Popular, estado y movimientos sociales. Una experiencia de gestión el frente de la SE-SP,1989-91. Argentina: 2a ed. Biblos:, 2019.

FREIRE, Paulo. FAÚNDEZ, Antonio. Por uma pedagogia da pregunta, Rio de Janeiro: Paz e Terra 1985.

FREIRE, Paulo. SHOR, Ira. Miedo y osadía, Argentina: Ed. Siglo XXI, 2014.

FREIRE, Paulo. Pedagogía de la ciudad. México: Ed. Siglo XXI, 2005.

GADOTTI, Moacir. Paulo Freire. Uma biografia, São Paulo: Cortez/Unesco, 2001.

GADOTTI, Moacir. CARNOY, Martin. Reinventando a Freire. São Paulo: Ed.IPF-Stanford Education, 2018.

LEVINSON, Jerome. ONIS, Juan. The Alliance That Lost Its Way, Chicago: Quadrangle Books, 1970.

MARTINS, Pablo. Confluencias entre el pensamiento de Franz Fanon y Paulo Freire. en Revista Educação. Santa Maria: v.37, n.2, maio/ago, 2018. p.241-256

PETERS, John. BELL, Brenda. 'Horton de Highlander' en vigésimo siglo pensadores en Educación de adultos, editado por Peter Jarvis, Routledge. 1987.

KOHAN, Walter. Paulo Freire mas que nunca. Una biografía filosófica, Buenos Aires: CLACSO, 2019:

SVAMPA, Maristella. La sociedad excluyente, La Argentina bajo el signo del neoliberalismo. Buenos Aires: Editorial Taurus, 2005.

ZIBECHI, Raúl. Genealogía de la revuelta. Argentina: La sociedad en movimiento. Buenos Aires: Editorial Letra Libre, 2003.

ZINN, Howard. La otra historia de los estados Unidos. Buenos Aires: Ed. Otras voces. 1999.

\section{FUENTES:}

-Documentos Highlander Center: https://www.highlandercenter.org/our-story

-Selección de documental, Instituto Paulo Freire, Sao Paulo.Brasil. 
-Nuñez García, Silvia y Zermeño Padilla, Guillermo (1988), EUA: Documentos de su historia política, T.III, Ed. Instituto Mora, México

Recebido em: 01 de Abril de 2020

Aceito para Publicação em 26 de Abril de 2020

(c) $\$(0)$ 